\title{
STAT3 inhibitor WP1066 attenuates miRNA-21 to suppress human oral squamous cell carcinoma growth in vitro and in vivo
}

\author{
XUAN ZHOU ${ }^{1,2^{*}}$, YU REN $^{3 *}$, AIQIN LIU $^{1 *}$, LEI HAN $^{4}$, KAILIANG ZHANG $^{4}$, SHASHA LI $^{1}$, \\ PENG LI ${ }^{5}$, PING LI ${ }^{1}$, CHUNSHENG KANG ${ }^{4}$, XUDONG WANG ${ }^{2}$ and LUN ZHANG ${ }^{1}$

\begin{abstract}
${ }^{1}$ Department of Otorhinolaryngology and Maxillofacial Oncology, Tianjin Medical University Cancer Institute and Hospital, National Clinical Research Center of Cancer, Tianjin 300060; ${ }^{2}$ Key Laboratory of Cancer Prevention and Therapy, Tianjin Cancer Institute, Tianjin 300060; ${ }^{3}$ Tianjin Research Center of Basic Medical Science, Tianjin Medical University, Tianjin 300070; ${ }^{4}$ Tianjin Medical University General Hospital, Laboratory of Neuro-Oncology, Tianjin Neurological Institute, Tianjin 300052; ${ }^{5}$ Tianjin Medical Information Institute, Tianjin 300070, P.R. China
\end{abstract}

Received December 5, 2013; Accepted March 4, 2014

DOI: 10.3892/or.2014.3114

\begin{abstract}
Abnormalities in signal transducer and activator of transcription 3 (STAT3) are involved in the oncogenesis of oral squamous cell carcinoma (OSCC). MicroRNA-21 (miR-21) is an important gene expression regulator to OSCC. miR-21 induction by STAT3 has been reported in multiple human cancers. In the present study, we found that STAT3 (-/p) expression was positively correlated with miR-21 in 60 OSCC samples. A reporter gene assay showed that miR-21 overexpression was dependent on STAT3 activation. WP1066, a small molecular inhibitor of STAT3, was used to suppress STAT3 expression in OSCC cells. TSCCA and TCA8113 showed reduction in tumor cell proliferation, invasion ability and miR-21 expression by WP1066 treatment. In addition, the expression of miR-21 target proteins [programmed cell death 4 (PDCD4), tissue inhibitor of metalloproteinase 3 (TIMP-3) and phosphatase and tensin homolog (PTEN)] was upregulated. Restored STAT3 expression by IL-6 induced miR-21 overexpression, which further confirmed the correlation between STAT3 and miR-21. WP1066 inhibited tumor growth and induced tumor cell apoptosis in the TSCCA xenograft tumor model. Western blotting and immunohistochemistry staining indicated that STAT3 (-/p), Ki67, Bcl-2 and MMP-2 expressions decreased in the WP1066-treated group;
\end{abstract}

Correspondence to: Professor Lun Zhang, Department of Otorhinolaryngology and Maxillofacial Oncology, Tianjin Medical University Cancer Institute and Hospital, Tianjin 300060, P.R. China E-mail: lun_zhang_jin@yahoo.com.cn

Professor Xudong Wang, Key Laboratory of Cancer Prevention and Therapy, Tianjin Cancer Institute, Tianjin 300060, P.R. China E-mail: wxd.1133@163.com

*Contributed equally

Key words: miRNA-21, signal transducer and activator of transcription 3, interleukin-6, WP1066, oral squamous cell carcinoma
PDCD4, TIMP-3 and PTEN expression increased simultaneously. The present study provides evidence that targeting STAT3 could regulate OSCC cell growth in a miR-21-dependent manner and WP1066 could be a novel candidate drug to treat OSCC by inhibiting STAT3/miR-21 axis.

\section{Introduction}

Head and neck squamous cell carcinoma (HNSCC) is one of the six most common types of cancer in the world, with $\sim 500,000$ new cases annually. Oral squamous cell carcinoma (OSCC) is the most common among the pathological types. The five-year survival rate after diagnosis for OSCC remains low at $\sim 50 \%$, which is considerably lower than that for other cancers, including colorectal, cervical and breast cancer $(1,2)$. To develop more optimized and more effective drug targets for OSCC treatment, a deeper understanding of the molecular mechanisms underlying oncogenesis and accurate identification of targets for therapeutic intervention are critical.

MicroRNAs (miRNAs) are classes of small, highly conserved non-coding RNAs that control gene expression by binding to the seed sequence at the 3 '-untranslated region (3'UTR) of targets, resulting in translational repression or mRNA degradation (3). miRNAs are regulators in human carcinogenesis, embryonic development and cell senescence. Previous studies have shown that the abundance of miRNAs is abnormally expressed in human cancer tissues, especially in the epithelium origin; for instance, miR-21 is characterized as an onco-miRNA (4). Several genes have been validated to be direct targets of miR-21 in human cancer. Programmed cell death 4 (PDCD4) is upregulated, whereas the glioblastoma cell is transfected with antisense miR-21 oligonucleotides and PDCD4's 3'UTR carrier binding sites of miR-21 (5). Phosphatase and tensin homolog (PTEN) are direct targets of miR-21, and reduction of miR-21 can suppress cancer cell proliferation via strengthened PTEN expression in hepatocellular carcinoma (6). In addition, miR-21 downregulates the tissue inhibitor of metalloproteinase-3 (TIMP-3) to influence cancer cell migration and invasion ability (7). 
Signal transducer and activator of transcription (STAT) proteins are the principal signaling proteins of cytokines and growth factors in mammals. STAT3 responds to signals, such as IL-6, IL-10 and VEGF. STAT3 is vital in tumorigenesis and cancer-induced immunosuppression $(8,9)$. Evidence has shown that STAT3 activates miR-21 to promote cancer cell growth (10-12). However, the precise biological function and molecular mechanism of STAT3/miR-21 network in OSCC remains unknown.

In the present study, we analyzed the correlation between miR-21 and STAT3 expression in OSCC samples. Results showed that the overexpression of miR-21 and STAT3 was related to lymph node metastasis and accumulative survival rate. We showed that blockage of STAT3 suppressed cancer cell growth and migration in OSCC, accompanying the increasing expressions of PDCD4, PTEN and TIMP-3 mediated by transcriptional inhibition of miR-21.

\section{Materials and methods}

Cell culture and reagents. Human TSCCA and TCA8113 OSCC cell lines were purchased from China Center for Type Culture Collection (Wuhan, China), and maintained in Dulbecco's modified Eagle's medium (DMEM) (Invitrogen, USA) supplemented with $10 \%$ fetal bovine serum (Gibco, USA), $2 \mathrm{mM}$ glutamine, 100 units of penicillin/ $\mathrm{ml}$ and $100 \mathrm{mg}$ of streptomycin $/ \mathrm{ml}$ (Sigma, USA) at $37^{\circ} \mathrm{C}$ with $5 \%$ $\mathrm{CO}_{2}$. WP1066 (Calbiochem, Germany) and IL-6 (Sigma) were dissolved in dimethyl sulfoxide (DMSO; Solarbo, China). WP1066 was added to cells at a final concentration of $5 \mu \mathrm{M}$ for 48-h treatment (10). IL-6-treated cells were referenced from a previous report (13).

Human tissue samples. The present study was approved by the Tianjin Medical University Institutional Review Board for human use. Ninety OSCC tissue samples were collected at the Department of Otorhinolaryngology and Maxillofacial Oncology at Tianjin Medical University Cancer Institute and Hospital. Tissue and clinical information were obtained as part of the study. Clinical diagnoses were reviewed to classify the pathological grade of the samples according to the American Joint Committee on Cancer TNM staging classification for the lip and oral cavity (2010). A total of 45 cases of high and moderate differentiated OSCC tumor samples, 45 cases of poorly differentiated OSCC tumor samples and 10 cancer adjacent relative normal margin tongue tissue samples were included in the current research. All the tumor and tumor margin tissues were paraffin-embedded for immunohistochemistry (IHC) staining and in situ hybridization.

miR-21 detection by in situ hybridization. Using antisense locked nucleic acid (LNA)-modified oligonucleotides probe, in situ hybridization was performed using an in situ hybridization kit (Boster, China). In situ hybridization detection of miR-21 in HNSCC samples and xenograft SCC sections was performed (14). LNA/DNA oligonucleotides contained locked nucleic acids at eight consecutive centrally located bases (indicated by the underlined part) and had the following sequences: LNA-miR-21, 5'-TCAACATCAGTCTGATAAGCTA-3'. Nuclei were counterstained using the DAPI karyotyping kit
(GenMed, USA) and were visualized using FluoView confocal laser scanning microscopes FV1000 (Olympus, Japan).

qRT-PCR analysis ofmiR-21 expression and RT-PCR for miR-21 targets. qRT-PCR analysis of miR-21 expression in OSCCcultured cells was performed (14). Total RNA was extracted by TRIzol (Invitrogen, USA). Both RT and PCR primers were purchased from GenePharma (Shanghai, China). U6 RNA was used for normalization. Relative quantification was conducted using amplification efficiencies derived from cDNA standard curves. Relative gene expressions were obtained. Quantitative analysis of change in expression levels was performed using a real-time PCR machine (7500 ABI; USA). MicroRNA Assay kit (Applied Biosystems) was used for detection of miR-21. The protocol was performed for 40 cycles at $95^{\circ} \mathrm{C}$ for $3 \mathrm{~min}, 95^{\circ} \mathrm{C}$ for $15 \mathrm{sec}$ and $60^{\circ} \mathrm{C}$ for $30 \mathrm{sec}$. Data were shown as fold-change $\left(2^{-\Delta \Delta \mathrm{Ct}}\right)$ and initially analyzed using Opticon Monitor analysis software version 2.02 (MJ Research, USA).

Exactly $5 \mu \mathrm{l}$ of the total $20 \mu \mathrm{l}$ of reverse-transcribed product was used for PCR $\left(30\right.$ cycles of $92^{\circ} \mathrm{C}$ for $15 \mathrm{sec}, 55^{\circ} \mathrm{C}$ for $30 \mathrm{sec}$ and $72^{\circ} \mathrm{C}$ for $2 \mathrm{~min}$ ) in $1 \mathrm{X}$ PCR buffer containing $1.5 \mathrm{mmol} / 1$ $\mathrm{MgCl}_{2}, 250 \mu \mathrm{mol} / 1$ deoxynucleotide triphosphates, 0.5 units of Taq polymerase (Life Technologies, USA), $100 \mathrm{ng}$ of primers for PDCD4 (forward primer, 5'-ATG GAT GTA GAA AAT GAG CAG-3' and reverse primer, 5'-TTA AAG TCT TCT CAA ATG CCC-3'); TIMP3 (forward primer, 5'-GAG AGT CTC TGT GGC CTT AAG CTG G-3' and reverse primer, 5'-CTG GGA AGA GTT AGT GTC CAA GGG-3'); PTEN (forward primer, 5'-ATT CCT CCA ATT CAG GAC CCA C-3' and reverse primer, 5'-CCT GGT ATG AAG AAC GTA TTT ACC C-3'), and GAPDH (forward primer, 5'-TCG TGG AAG GAC TCA TGA CC-3' and reverse primer, 5'-TCC ACC ACC CTG TTG CTG TA-3') (SBS Bio, Beijing, China). The reaction products were analyzed on $2 \%$ agarose gels containing ethidium bromide; cDNA synthesis was verified by detection of $\beta$-actin transcript.

Cancer cell clone formation, cell cycle, apoptosis and Transwell chamber assays. Cancer cell clone formation, cell cycle, apoptosis and Transwell chamber assays were performed as described before (15).

Luciferase assay. The pMIR-21-Report-Luc reporter plasmid was purchased from Signosis (Signosis, USA). The mature miR-21 binding sequence was subcloned into a firefly luciferase-based reporter construct immediately downstream of Luc coding sequence. TSCCA and TCA8113 cells were plated $\left(2 \times 10^{6}\right.$ cells/well) in 6-well plates. After transfection, the cells were split into 96 -well plates $\left(4 \times 10^{3}\right.$ cells/well) in duplicate and harvested for luciferase assays $24 \mathrm{~h}$ later using a Luciferase Assay kit (Promega, USA).

Western blot analysis. Parental and WP1066-treated cells were washed with ice-cold phosphate-buffered saline (PBS) three times. The cells were solubilized in $1 \%$ Nonidet P-40 lysis buffer $(20 \mathrm{mM}$ Tris, $\mathrm{pH} 8.0,137 \mathrm{mM} \mathrm{NaCl}, 1 \%$ Nonidet P-40, $10 \%$ glycerol, $1 \mathrm{mM} \mathrm{CaCl} 2,1 \mathrm{mM} \mathrm{MgCl}_{2}$, $1 \mathrm{mM}$ phenylmethylsulfonyl fluoride, $1 \mathrm{mM}$ sodium fluoride, $1 \mathrm{mM}$ sodium orthovanadate and a protease inhibitor mixture). A total of $40 \mathrm{mg}$ lysates were subjected to SDS-PAGE on 

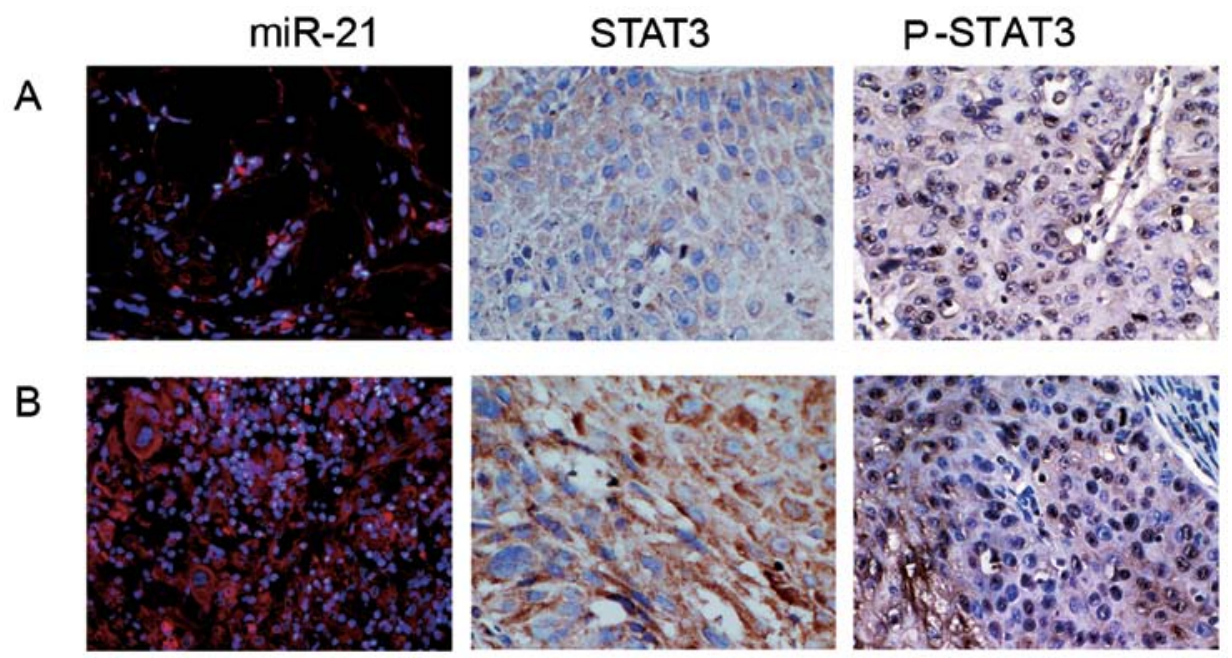

Figure 1. miR-21, STAT3 and p-STAT3 expressions in OSCC tissue samples. (A) In highly differentiated OSCC tumors, in situ hybridization staining revealed that miR-21 (red) has a relatively low expression in tumor cell cytoplasm (original magnification, x100); IHC staining showed that STAT3 (brown signal in tumor cell cytoplasm and nucleus) and p-STAT3 (brown signal in tumor cell nucleus) have low expressions (original magnification, x200). (B) In moderately and poorly differentiated OSCC tumors, in situ hybridization staining showed the high expression of miR-21 in the cytoplasm (original magnification, $\mathrm{x} 100$ ); IHC staining showed that STAT3 and p-STAT3 have relatively high expressions (original magnification, $\mathrm{x} 200$ ).

$8 \%$ SDS-acrylamide gel. Separate proteins were transferred to PVDF membranes (Millipore, USA) and incubated with primary antibodies against STAT3, p-STAT3, PTEN, PDCD4, TIMP-3, Ki67, MMP2 and Bcl-2 (Santa Cruz, USA), followed by incubation with HRP-conjugated secondary antibody (Zymed, USA). GAPDH (Santa Cruz) was used as internal control. The specific protein was detected using a SuperSignal protein detection kit (Pierce, USA).

TSCCA xenograft tumor assay. Nude mouse subcutaneous TSCCA xenograft model was performed as described before (14). The mice were randomly divided into three groups when tumors reached $\sim 10 \mathrm{~mm}$ in length. Two groups of mice were treated with DMSO (local injection, once every 3 days for 21 days) or WP1066 (40 mg/kg, local injection) alone. One group of mice was taken as control. The tumor volume was measured with a caliper every 3 days using the formula: Volume $=$ length $\mathrm{x}$ width $/ 2$. At the end of the observation period, the mice bearing xenograft tumors were sacrificed and the tumor tissues were removed for formalin fixation and preparation of paraffin-embedded sections.

IHC staining. The paraffin-embedded tissue sections were used for examination of STAT3, p-STAT3,PTEN, PDCD4, TIMP-3, $\mathrm{Ki} 67, \mathrm{MMP} 2$ and $\mathrm{Bcl}-2$ expressions, as well as HE staining. For IHC assay, sections were incubated with primary antibodies (1:200 dilutions) overnight at $4^{\circ} \mathrm{C}$, followed by a biotin-labeled secondary antibody (1:100 dilutions) for $1 \mathrm{~h}$ at room temperature. Sections were incubated with ABC-peroxidase and diaminobenzidine (DAB), counterstained with hematoxylin and visualized using light microscopy.

TUNEL assay. Apoptotic cell death in the tumor specimens of mouse models was examined by the TUNEL method using an in situ cell death kit (Roche, USA). Positive cells were visualized using fluorescence microscopy. The reaction mixture was incubated without enzyme in a control coverslip to detect non-specific staining. Nuclei were counterstained with DAPI karyotyping kit and visualized using FV-1000 laser scanning confocal biological microscopes and analyzed using IPP5.1 (Olympus).

Statistical analysis. Results are presented as averages of at least three experiments performed in triplicate with respective standard errors. Statistical evaluation for data analysis was determined by $\mathrm{t}$-test and $\chi^{2}$ test; differences with $\mathrm{P}<0.05$ were considered statistically significant.

\section{Results}

STAT3 expression correlates with miR-21 in OSCC tissue samples. miR-21 is overexpressed in OSCC, including cultured cell lines and tumor tissue samples $(16,17)$. In the present study, we assessed miR-21 expression in 90 OSCC tissue samples with distinct differentiation status to study the regulatory mechanism of miR-21 in OSCC oncogenesis. Using an LNA-modified antisense miR-21 probe, miR-21 expressions in well-differentiated (positive rate, $45.0 \pm 15.2$ ), moderately differentiated (positive rate, $71.7 \pm 17.1$ ) and poorly differenti-

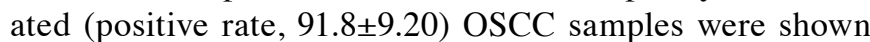
(Fig. 1). IHC staining indicated that positive STAT3 signal resided in both nucleus and cytoplasm. The STAT3 expression rates for poorly differentiated, moderately differentiated, and well-differentiated OSCC samples were: $89.2 \pm 14.5,68.1 \pm 20.2$ and $46.3 \pm 12.5$, respectively (Fig. 1). Pearson's rank correlation analysis showed a high, positive correlation $(\mathrm{R}=0.902, \mathrm{P}<0.05)$ between STAT3 and miR-21 expressions among different OSCC samples.

Reduction of STAT3 by WP1066 inhibits OSCC growth in vitro. We employed WP1066, a specific STAT3 small-molecule inhibitor, to suppress STAT3 activity in OSCC cancer cells. Calculation of cell clone numbers revealed that TSCCA and TCA8113 cells exhibited more decreased proliferation 

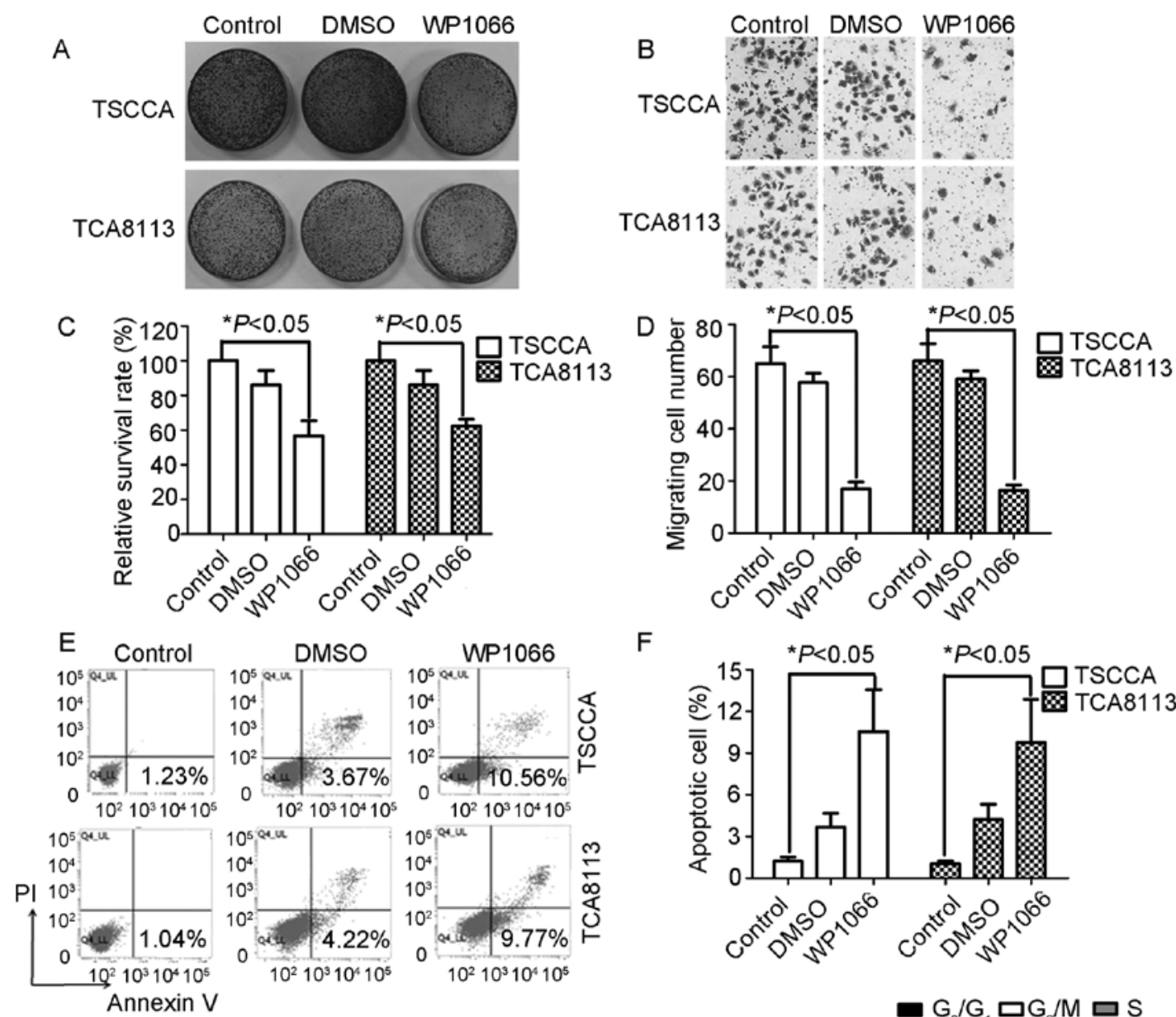

$\mathrm{F}$
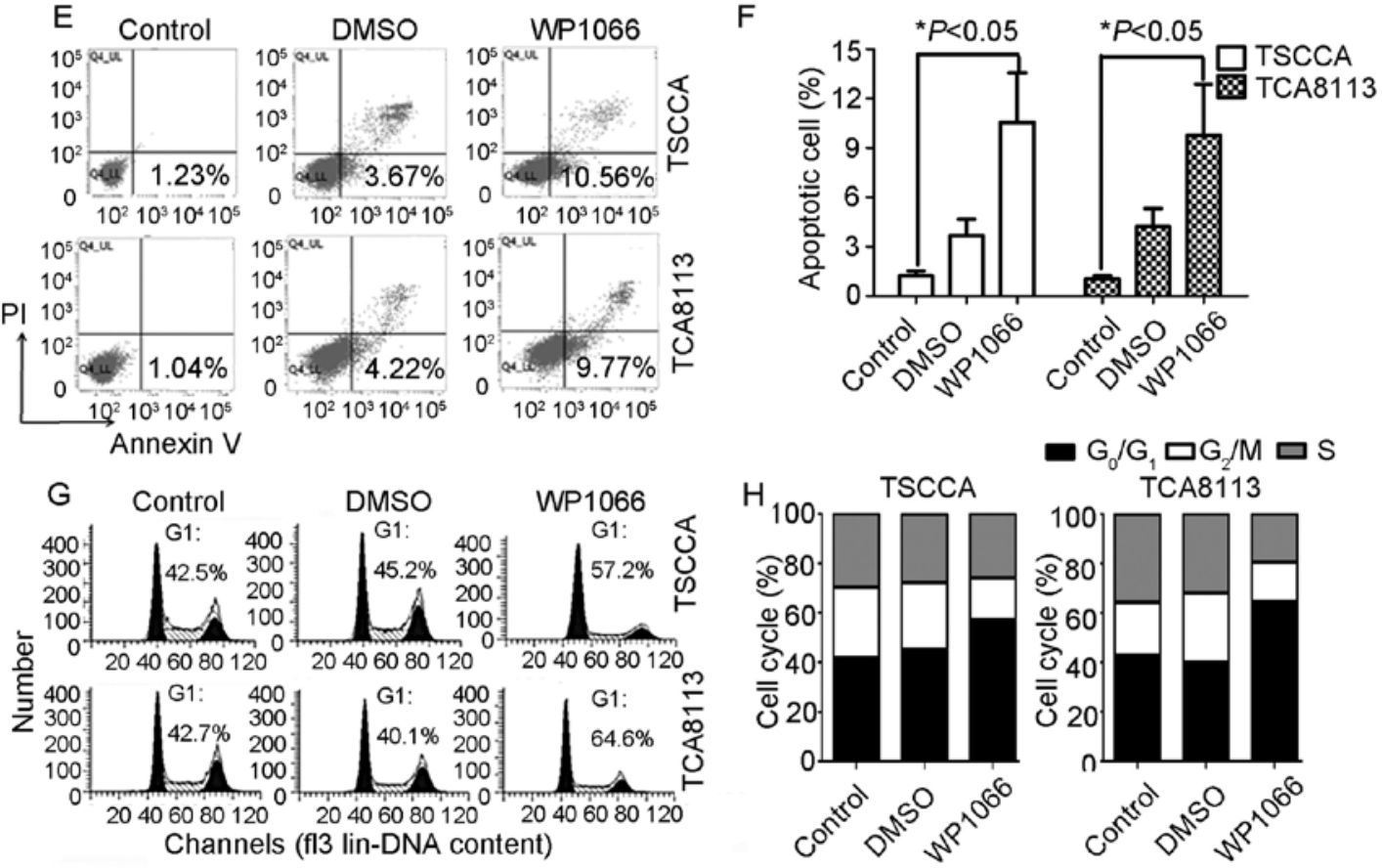

Figure 2. Reduction of STAT3 by WP1066 inhibits OSCC growth in vitro. (A and B) Reduction of clone formation ability in WP1066-treated TSCCA and TCA8113 cells from the colony formation assay. (C and D) Transwell assay showed migration ability reduction in WP1066-treated TSCCA and TCA8113 cells. (E and F) Early apoptotic nucleus was shown in WP1066-treated TSCCA and TCA8113 cells using FCM examination. (G and H) FCM examination revealed a cycle arrest in WP1066-treated TSCCA and TCA8113 cells at G1 phase.

ability than control and DMSO-treated groups following treatment with WP1066. The survival rate of WP1066-treated TSCCA cells was 56.67\% ( $\mathrm{F}=19.99, \mathrm{P}=0.002$; Fig. 2A and C), and the survival rate of WP1066-treated TCA8113 cells was $62.33 \%(\mathrm{~F}=61.81, \mathrm{P}=0.000)$. Transwell assay was employed to measure STAT3's effects on OSCC in vitro cell invasiveness. The number of invasive cells in WP1066-treated cultures was reduced relative to the control cells. Fig. $2 \mathrm{~B}$ and D show reduction from $65.00 \pm 11.14$ to $17.00 \pm 4.58$ in TSCCA cells and $66.00 \pm 11.53$ to $16.33 \pm 3.79$ in TCA8113 cells. Transwell assay indicated that the invasiveness of cancer cells and the migration ability were significantly attenuated through inhibition of STAT3 activity. The effect of decreased STAT3 on apoptosis was analyzed through Annexin V and PI double staining. The
Annexin V-positive early-phase apoptotic cells significantly increased in WP1066-treated cells $(10.56 \%$ for TSCCA cells and $9.77 \%$ for TCA8113 cells) when compared with parental and DMSO-treated cells ( $\mathrm{P}=0.01$; Fig. $2 \mathrm{E}$ and $\mathrm{F})$. The early-phase apoptosis data were consistent with the cell cycle blockage in both cell lines. G1 phase percentage of TSCCA cells was $57.2 \%$, a value that was $14.7 \%$ higher than that in the control group, $72 \mathrm{~h}$ following WP1066 treatment. Meanwhile, G1 phase percentage of TCA8113 cells was $64.6 \%$, a value that was $21.9 \%$ higher than that in the control group. Both TSCCA and TCA8113 cells displayed G1 phase blockage (Fig. 2G and H). Western blot analysis indicated that MMP-2 expression was inhibited effectively in WP1066-treated TSCCA and TCA8113 cells, Ki-67 and Bcl-2 (Fig. 4A). 
A

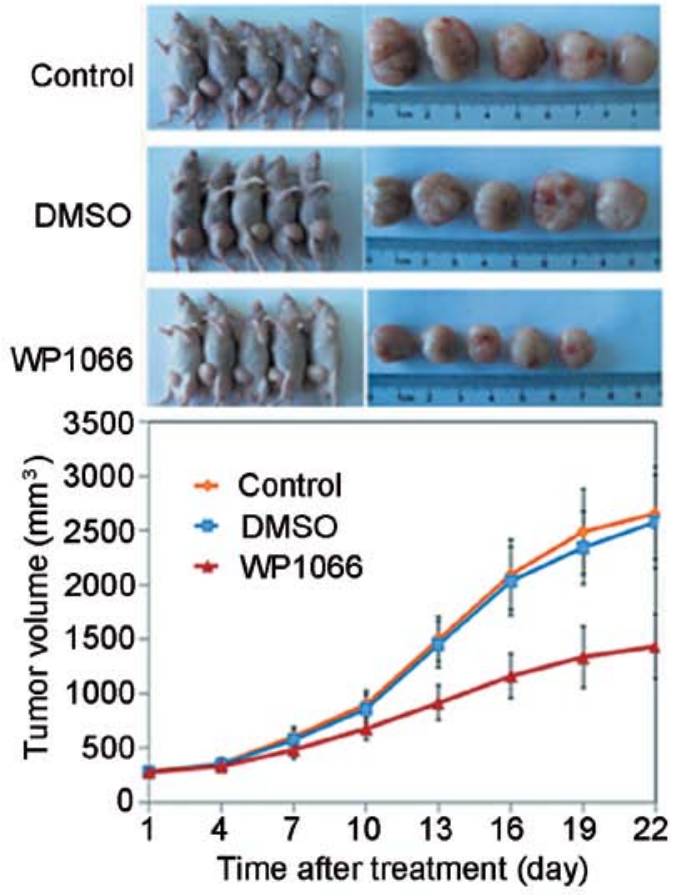

B

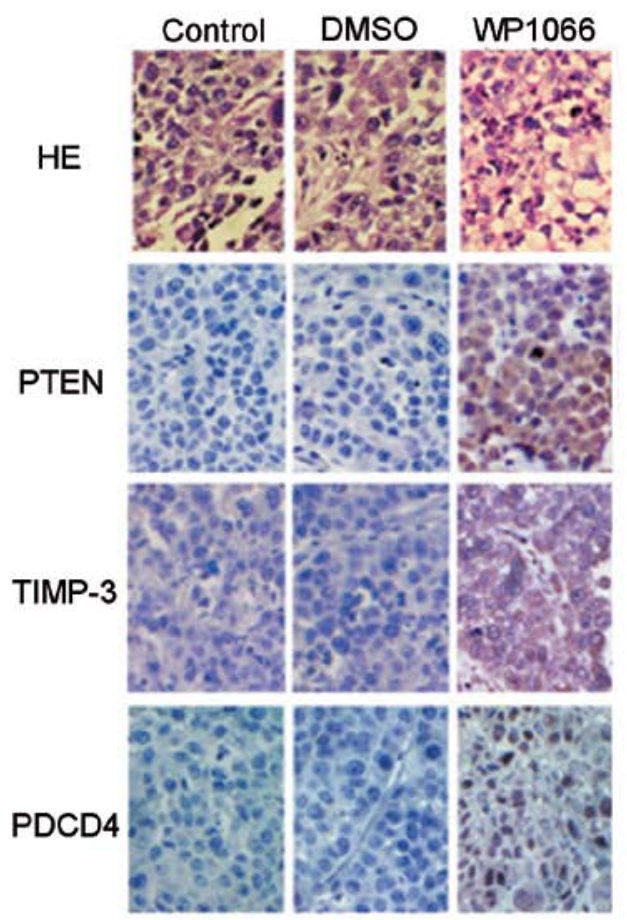

D
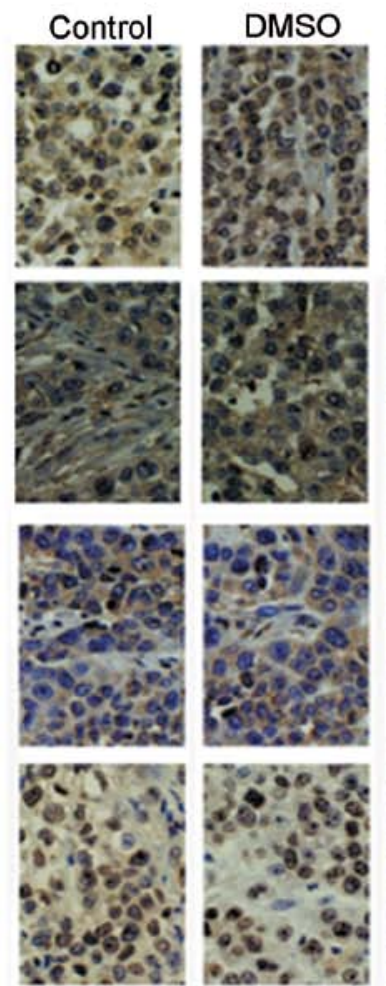

WP1066
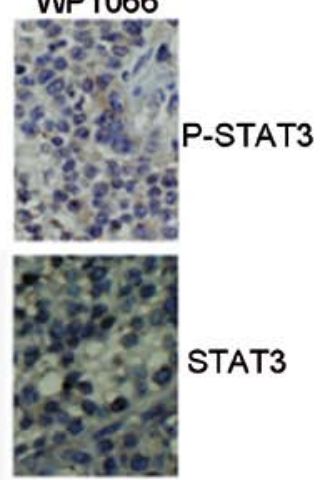

STAT3
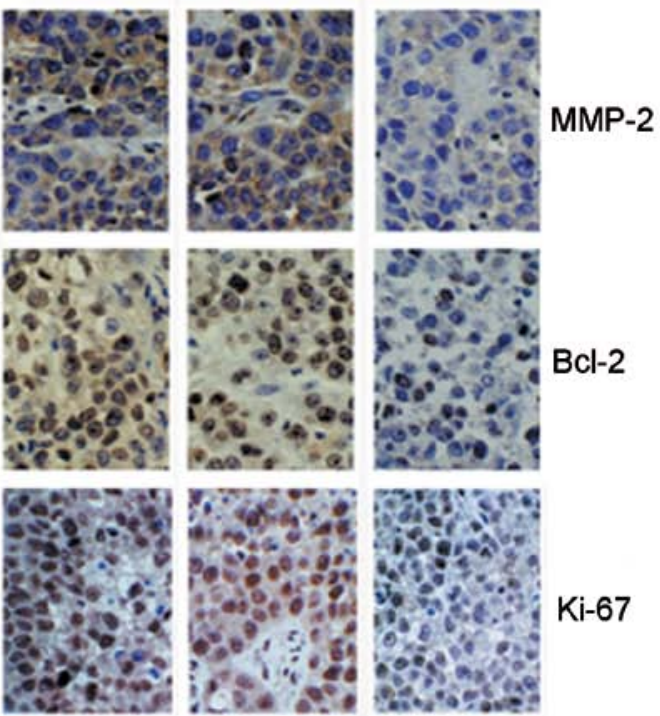

E
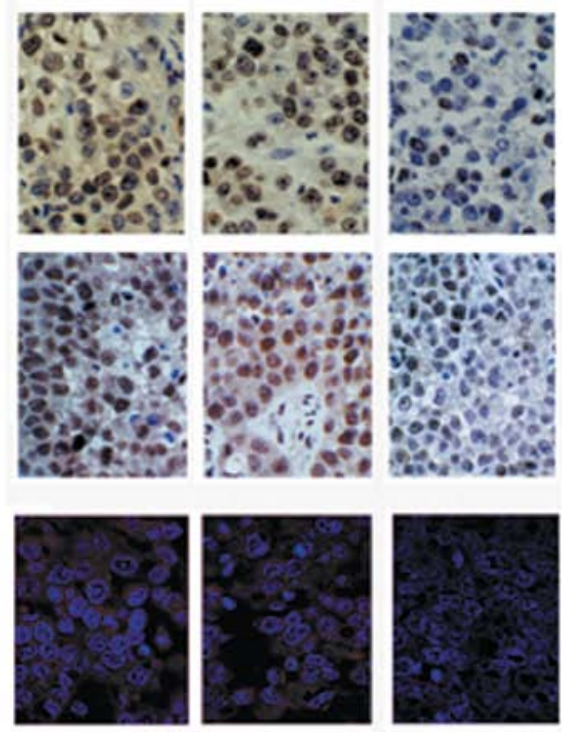

$\mathrm{Bcl}-2$

F
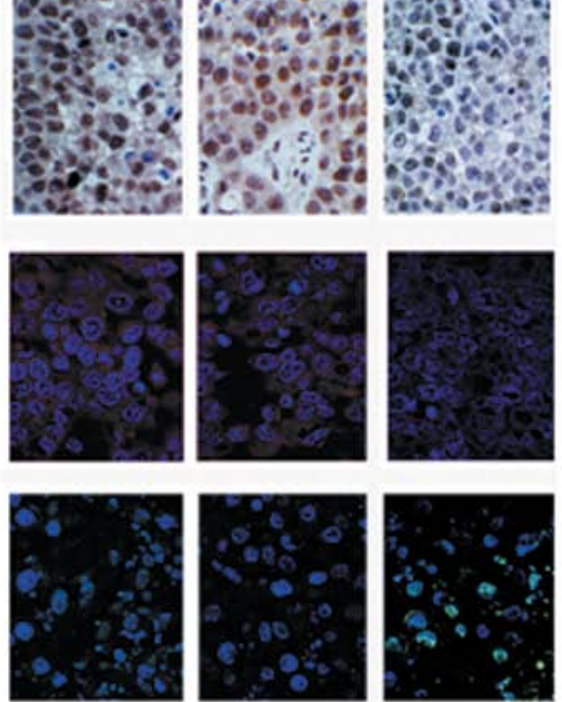

Figure 3. Inhibition of in vivo TSCCA cell growth by reduction of STAT3. (A) Image and growth curves of control, DMSO-treated and WP1066-treated TSCCA xenograft tumors. (B) HE staining of TSCCA xenograft tumors (original magnification, $x 200$ ). (C) IHC staining showed the upregulation of miR-21 expressions of target proteins (PTEN, TIMP-3 and PDCD4; original magnification, x200) in the WP1066-treated group. (D) Downregulation of the expressions of STAT3 (-/p), Ki67, Bcl-2 and MMP-2 in WP1066-treated group (original magnification, x200) was observed using IHC staining. (E) Downregulation of miR-21 expression (red) in the WP1066-treated group from in situ hybridization staining (original magnification, x200). (F) TUNEL assay showed an induced apoptotic nucleus (green) in WP1066-treated TSCCA tumors (original magnification, x200).

WP1066 suppresses TSCCA growth in a xenograft model. In vitro experiments indicated that STAT3 was a potential target in OSCC therapy. We performed a proof-of-principle experiment using a TSCCA cell xenograft model and a DMSO-diluted WP1066 therapy approach for confirmatory purposes. Ten mice were challenged by in situ injection of DMSO as a negative control group, and another 10 mice were treated with PBS as a control group. The mean tumor volume of the mice in the control, the DMSO and the WP1066-treated groups was $\sim 280 \mathrm{~mm}^{3}$, with no statistically significant difference at the onset of the treatment. The mice were monitored and treated every 3 days for 3 weeks, and the tumor volume of the mice in each group was measured. Fig. 3A shows that a significant decrease in tumor volume was only observed in the WP1066-treated group $(\mathrm{F}=15.390$, $\mathrm{P}=0.000)$. 
A

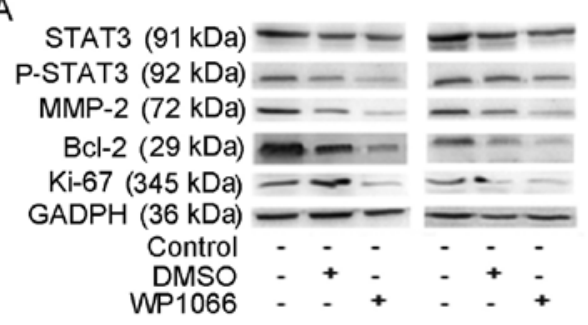

C

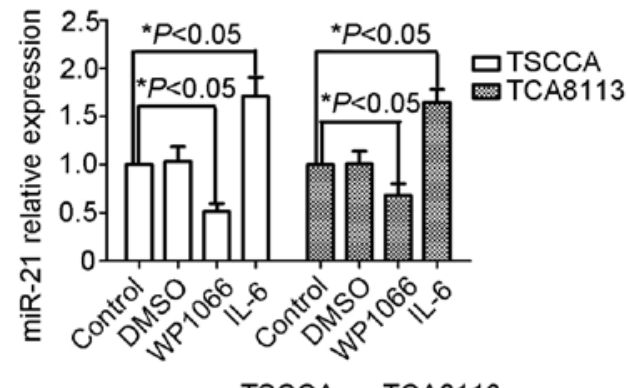

E

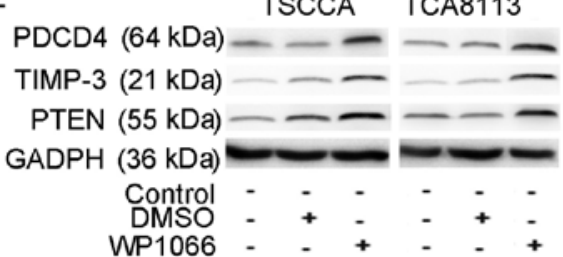

G
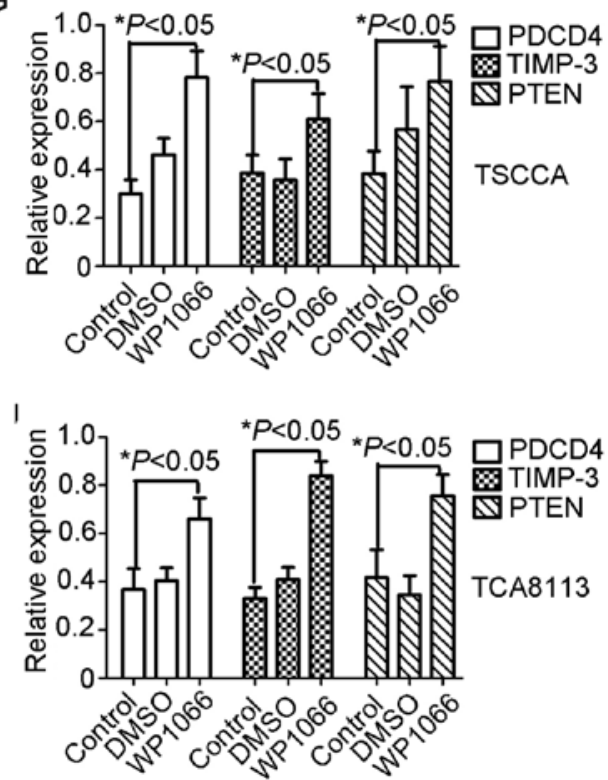

B

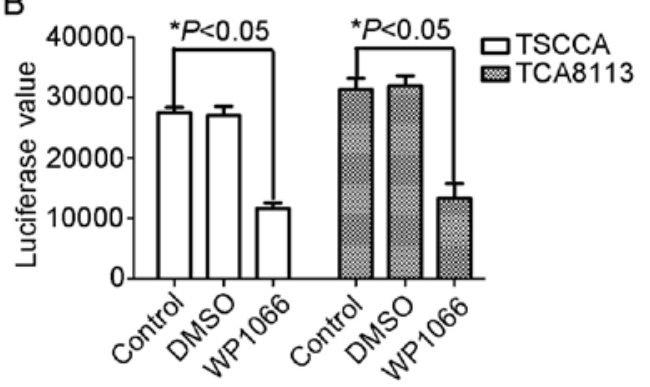

F

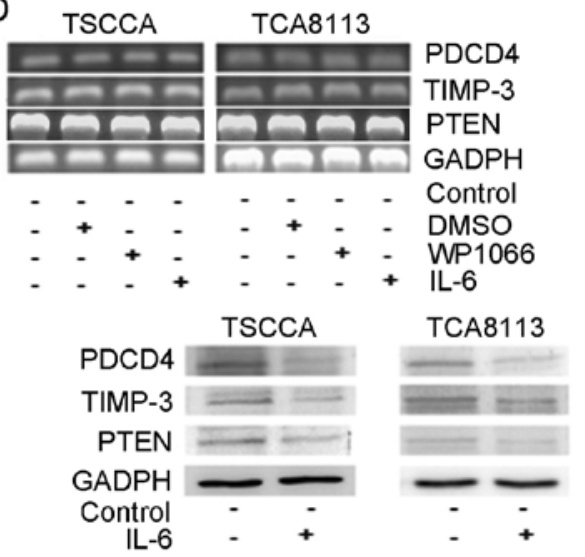

$\mathrm{H}$
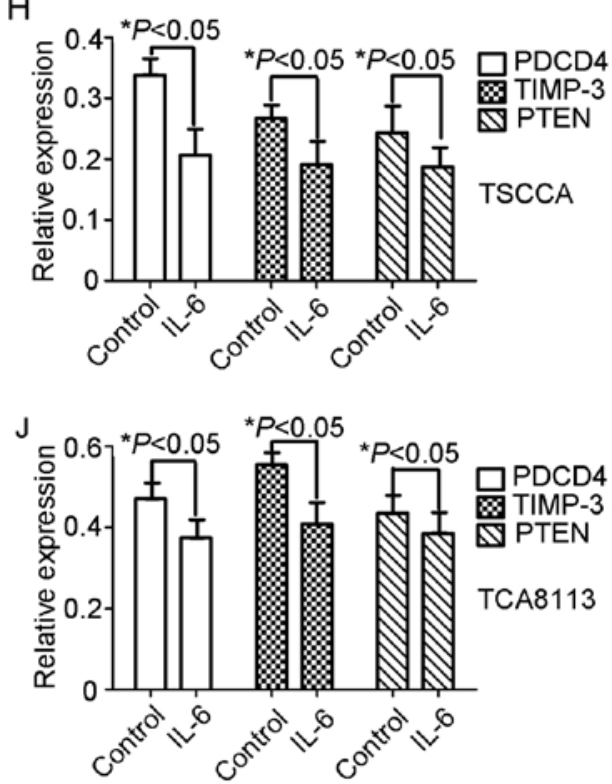

Figure 4. WP1066 inhibits miR-21 expression in OSCC. (A) STAT3 (-/p), Ki67, Bcl-2 and MMP-2 expressions were downregulated in WP1066-treated TSCCA and TCA8113 cells. (B) pMIR-21-Report-Luc reporter plasmid assay indicated that the miR-21 gene promoter carries a STAT3 binding site. (C) Inhibition of miR-21 expression in WP1066-treated TSCCA and TCA8113 cells, and that of TSCCA and TCA8113 cells treated with IL-6 from Q-PCR. (D) WP1066 and IL-6 had no influence on PDCD4, TIMP-3 and PTEN mRNA level, based on RT-PCR. (E, G and I) WP1066 induced PDCD4, TIMP-3 and PTEN protein expressions in TSCCA and TCA8113 cells. (F, H and J) IL-6 inhibited PDCD4, TIMP-3 and PTEN protein expressions in TSCCA and TCA8113 cells.

HE stain was revealed in WP1066-treated tumor samples. Atypia tumor cells and necrosis cells reduced in number and in size. Tumor tissue derived from WP1066-treated groups showed decreased microvessel density and chromatin staining compared with those from the control groups (Fig. 3B). TUNEL staining assay data showed that more apoptotic nuclei were found in the WP1066-treated group, revealing that STAT3 inhibition caused tumor cell apoptosis and contributed to tumor growth suppression of the xenografts (Fig. 3F).
STAT3 and p-STAT3 expressions were inhibited significantly (Fig. 3D), and miR-21 expression was decreased in xenograft tumors (Fig. 3E) for the WP1066-treated group. Thus, STAT3-miR-21 adhered to an in vivo regulatory mechanism. We observed decreased Ki-67, MMP-2 and Bcl-2 expressions, consistent with in vitro results, in the WP1066-treated tumors (Fig. 3D). We measured the expression status of miR-21 in the animal model. Fig. 3C shows an increase of PTEN, PDCD4 and TIMP-3 from the IHC staining data. 
WP1066 inhibits miR-21 expression in OSCC. We generated a pMIR-21-Report-Luc reporter plasmid containing the putative STAT3 binding sites to miR-21 promoter in an attempt to address the stable relationship between STAT3 and miR-21 in oral cancer cell lines. The pMIR-21-Report-Luc reporter construct was separately co-transfected with WP1066 or DMSO into TSCCA and TCA8113 cells, followed by measurement of luciferase activity in the transfected cells 3 days after transfection. Results showed that WP1066 caused 50-60\% decrease in luciferase activity (Fig. 4B), indicating that STAT3 promotes miR-21 gene transcription.

We examined the expression changes in the biological direct targets of miR-21 (PDCD4, PTEN and TIMP-3) at mRNA and protein levels. RT-PCR results indicated no significant difference in PDCD4, PTEN and TIMP-3 mRNA expression levels among the control, DMSO-treated and WP1066-treated TSCCA and TCA8113 cell groups (Fig. 4D). The protein levels of PDCD4, PTEN and TIMP-3 increased following treatment with WP1066 in both cell lines (Fig. 4E, G and I) compared with the control and the DMSO-treated groups.

To further illustrate the hypothesized STAT3-miR-21 regulatory axis, we enrolled IL-6 to upregulate STAT3 activity and to examine miR-21 and biological target expressions. miR-21 expression increased in IL-6-treated TSCCA and TCA8113 cells (Fig. 4C). PDCD4, PTEN and TIMP-3 expressions exhibited decreased protein levels, but no significant changes were found in the mRNA levels (Fig. 4D and E). These data provide additional evidence of the influence of STAT3 on miR-21 expression at the transcription level.

\section{Discussion}

Previous studies have reported on the activation of STAT family and the control of aberrantly expressed miRNAs in the most basic mechanisms of oral squamous cell carcinoma (OSCC) $(19,20)$. In the present study, we showed that miR-21 and STAT3 were more co-overexpressed in human OSCC tissue samples compared with tumor-adjacent tissues. miR-21 is one of the most overexpressed miRNAs in a number of medium-scale and large-scale profiling experiments designed to detect dysregulated miRNAs in human cancers (21). Overexpression of miR-21 could increase cell proliferation, migration, invasion and survival, as confirmed in breast cancer, glioma, hepatocellular carcinoma, OSCC, myeloma and colon cancer (4). Systemic review and meta-analysis have shown that miR-21 detection has a prognostic value in cancer patients, especially in HNSCC and gastrointestinal cancers. Higher expression of miR-21 has been correlated with overall survival in HNSCC and the combined HR is 1.46 (22). Data suggested that miR-21 is a candidate biomarker for cancer treatment; thus, the mechanism of elevated expression of miR-21 in human cancers should be studied in the future.

Several studies have established that miR-21 is induced by diverse mechanisms, such as genetic alterations, mutations, single nucleotide polymorphisms, Drosha and Dicer activity, methyltransferases and histone deacetylases (23). For instance, Toll ligand receptor activation by LPS upregulates miR-21 in numerous cells types, including macrophages, fibroblasts and peripheral blood mononuclear cells (24). IL-6 promotes the survival of multiple myeloma cells through induction of
miR-21 expression by STAT3 activation (25). In situ hybridization and IHC data indicated a linear correlation expression mechanism of STAT3 and miR-21 gene putative promoter region. We employed WP1066, a specific STAT3 inhibitor, to suppress STAT3 activation in TSCCA and TCA8113 cells (26). In the OSCC cell lines and animal model, miR-21 expression downregulated linearly upon treatment with WP1066. Tumor suppressor genes, such as PTEN, TIMP-3 and PDCD4, are functional targets of miR-21 (5-7). STAT3 inhibition triggered upregulation of protein expression levels but not mRNA levels, and IL-6 induced STAT3 activation could inhibit these gene expressions. Luciferase assay suggested that the miR-21 promoter region contained a STAT3 putative regulatory sequence. Data suggested the existence of a STAT3 $\rightarrow$ miR-21 transcriptional regulatory axis in OSCC.

Early studies indicated that HNSCC and the derived cell lines exhibit markedly elevated levels of phosphorylated STAT3 (29) among the STAT protein family members, whereas constitutive activation of STAT3 has been demonstrated in many types of cancer, including breast, lung and thyroid cancer $(27,28)$. Increased STAT3 levels alter cell cycle progression and promote the proliferation and survival of tumor cells (30). Both in vitro and in vivo treatment of WP1066 in OSCC cells showed significant reduction of cancer cell clone formation ability, increased apoptotic nuclei, cancer cell migration, invasion inhibition and cell cycle blockage at G1 phase. We inferred that miR-21 reduction brought by WP1066 treatment could partially explain the mechanism of the results. PTEN expression increased and the AKT signaling pathway was suppressed due to the downregulation of miR-21 in OSCC cells. The AKT pathway is responsible for cancer survival and metastasis by regulating Bcl-2 and MMP2/9 (31). TIMP3, the ECM-bound protease regulator, are key inhibitors of several MMPs and the expression is prognostic for cancer (32). Thus, the recovery of TIMP-3 protein is responsible for the migration and reduction of invasion in OSCC cells. PDCD4 reportedly inhibits AP-1-mediated trans-activation and induces expression of the cyclin-dependent kinase inhibitor p21 (33). Thus, the OSCC cell cycle blockage in G1 phase occurred due to the upregulation of PDCD4.

Our results provide evidence of the inhibition of STAT3 by WP1066, resulting in upregulation of PTEN, PDCD4 and TIMP-3 by suppression of miR-21, as well as the contribution of STAT3/miR-21 pathway in in vitro and in vivo OSCC growth. In addition, WP1066 could be a novel candidate drug to treat OSCC by inhibiting STAT3/miR-21 axis.

\section{Acknowledgements}

This study was supported by the China National Natural Scientific Fund (grant numbers 81172573, 81101916 and 51103107), and the Tianjin Science and Technology Committee (grant numbers 11JCYBJC10800 and 12JCYBJC33800).

\section{References}

1. Posner M and Vermorken JB: Induction therapy in the modern era of combined-modality therapy for locally advanced head and neck cancer. Semin Oncol 35: 221-228, 2008.

2. Cohen EE, Lingen MW and Vokes EE: The expanding role of systemic therapy in head and neck cancer. J Clin Oncol 9: $1743-1752,2004$ 
3. He L and Hannon GJ: MicroRNAs: small RNAs with a big roll in gene regulation. Nat Rev Genet 5: 522-531, 2004.

4. Pan X, Wang ZX and Wang R: MicroRNA-21: a novel therapeutic target in human cancer. Cancer Biol Ther 12: 1224-1232, 2010.

5. Gaur AB, Holbeck SL, Colburn NH and Israel MA: Downregulation of Pdcd4 by mir-21 facilitates glioblastoma proliferation in vivo. Neuro Oncol 13: 580-590, 2011.

6. Meng F, Henson R, Wehbe-Janek H, et al: MicroRNA-21 regulates expression of the PTEN tumor suppressor gene in human hepatocellular cancer. Gastroenterology 133: 647-658, 2007.

7. Gabriely G, Wurdinger T, Kesari S, et al: MicroRNA 21 promotes glioma invasion by targeting matrix metalloproteinase regulators. Mol Cell Biol 28: 5369-5380, 2008.

8. Rawlings JS, Rosler KM and Harrison DA: The JAK/STAT signaling pathway. J Cell Sci 117: 1281-1283, 2004.

9. He G and Karin M: NF- $\mathrm{B}$ and STAT3 - key players in liver inflammation and cancer. Cell Res 21: 159-168, 2011.

10. Wang YY, Sun G, Luo H, et al: MiR-21 modulates hTERT through a STAT3-dependent manner on glioblastoma cell growth. CNS Neurosci Ther 18: 722-728, 2012.

11. Han L, Yue X, Zhou X, et al: MicroRNA-21 expression is regulated by $\beta$-catenin/STAT3 pathway and promotes glioma cell invasion by direct targeting RECK. CNS Neurosci Ther 18 573-583, 2012.

12. Yang $\mathrm{CH}$, Yue J, Pfeffer SR, et al: MicroRNA miR-21 regulates the metastatic behavior of B16 melanoma cells. J Biol Chem 286 39172-39178, 2011.

13. Lederle W, Depner S, Schnur S, et al: IL-6 promotes malignant growth of skin SCCs by regulating a network of autocrine and paracrine cytokines. Int J Cancer 128: 2803-2814, 2011.

14. Zhou X, Ren Y, Moore L, et al: Downregulation of miR-21 inhibits EGFR pathway and suppresses the growth of human glioblastoma cells independent of PTEN status. Lab Invest 90: 144-155, 2010

15. Han L, Zhang A, Wang H, et al: Tat-BMPs-PAMAM conjugates enhance therapeutic effect of small interference RNA on U251 glioma cells in vitro and in vivo. Hum Gene Ther 4: 417-426, 2010.

16. Li J, Huang H, Sun L, et al: MiR-21 indicates poor prognosis in tongue squamous cell carcinomas as an apoptosis inhibitor. Clin Cancer Res 15: 3998-4008, 2009.

17. Yu ZW, Zhong LP, Ji T, et al: MicroRNAs contribute to the chemoresistance of cisplatin in tongue squamous cell carcinoma lines. Oral Oncol 46: 317-322, 2010.

18. Parkin DM, Bray F, Ferlay J, et al: Global cancer statistics, 2002. CA Cancer J Clin 55: 74-108, 2005.
19. Grandis JR, Drenning SD, Zeng Q, et al: Constitutive activation of Stat 3 signaling abrogates apoptosis in squamous cell carcinogenesis in vivo. Proc Natl Acad Sci 97: 4227-4232, 2000.

20. Bourguignon LY, Earle C, Wong G, et al: Stem cell marker (Nanog) and Stat-3 signaling promote MicroRNA-21 expression and chemoresistance in hyaluronan/CD44-activated head and neck squamous cell carcinoma cells. Oncogene 31: 149-160, 2012.

21. Volinia S, Calin GA, Liu CG, et al: A micro-RNA expression signature of human solid tumors defines cancer gene targets. Proc Natl Acad Sci USA 103: 2257-2261, 2006.

22. Fu X, Han Y, Wu Y, et al: Prognostic role of microRNA-21 in various carcinomas: a systematic review and meta-analysis. Eur J Clin Invest 41: 1245-1253, 2011.

23. Iorio MV andCroce CM: Causes and consequences of microRNA dysregulation. Cancer J 18: 215-222, 2012.

24. Sheedy FJ, Palsson-McDermott E, Hennessy EJ, et al: Negative regulation of TLR4 via targeting of the proinflammatory tumor suppressor PDCD4 by the microRNA miR-21. Nat Immunol 11: $141-147,2010$.

25. Löffler D, Brocke-Heidrich K, Pfeifer G, et al: Interleukin-6 dependent survival of multiple myeloma cells involves the Stat3mediated induction of microRNA-21 through a highly conserved enhancer. Blood 110: 1330-1333, 2007.

26. Horiguchi A, Asano T, Kuroda K, et al: STAT3 inhibitor WP1066 as a novel therapeutic agent for renal cell carcinoma. $\mathrm{Br}$ J Cancer 102: 1592-1599, 2010.

27. Darnell JE: Validating Stat3 in cancer therapy. Nat Med 11: 595-596, 2005.

28. Bromberg J: Stat proteins and oncogenesis. J Clin Invest 109: 1139-1142, 2002

29. Grandis JR, Drenning SD, Chakraborty A, et al: Requirement of Stat3 but not Stat1 activation for epidermal growth factor receptor-mediated cell growth in vitro. J Clin Invest 102: 1385-1392, 1998

30. Johnson DE: Targeting proliferation and survival pathways in head and neck cancer for therapeutic benefit. Chin J Cancer 31: 319-326, 2012.

31. Agarwal E, Brattain MG and Chowdhury S: Cell survival and metastasis regulation by Akt signaling in colorectal cancer. Cell Signal 25: 1711-1719, 2013.

32. Kotzsch M, Farthmann J, Meye A, et al: Prognostic relevance of uPAR-del4/5 and TIMP-3 mRNA expression levels in breast cancer. Eur J Cancer 41: 2760-2768, 2005.

33. Göke R, Barth P, Schmidt A, et al: Programmed cell death protein 4 suppresses CDK1/cdc2 via induction of $\mathrm{p} 21^{\text {Waf1/Cip1 }}$. Am J Physiol Cell Physiol 287: C1541-C1546, 2004. 